\title{
Power Allocation Scheme for Femto-to-Macro Downlink Interference Reduction for Smart Devices in Ambient Intelligence
}

\author{
Xin Su, ${ }^{1}$ Chengchao Liang, ${ }^{2}$ Dongmin Choi, ${ }^{3}$ and Chang Choi ${ }^{4}$ \\ ${ }^{1}$ College of IoT Engineering, Changzhou Key Laboratory of Robotics and Intelligent Technology, Hohai University, Changzhou, China \\ ${ }^{2}$ Department of Systems and Computer Engineering, Carleton University, Ottawa, ON, Canada \\ ${ }^{3}$ Division of Undeclared Majors, Chosun University, Gwangju, Republic of Korea \\ ${ }^{4}$ Department of Computer Engineering, Chosun University, Gwangju, Republic of Korea
}

Correspondence should be addressed to Chang Choi; enduranceaura@gmail.com

Received 1 October 2016; Accepted 28 November 2016

Academic Editor: Francesco Palmieri

Copyright (C) 2016 Xin Su et al. This is an open access article distributed under the Creative Commons Attribution License, which permits unrestricted use, distribution, and reproduction in any medium, provided the original work is properly cited.

In this paper, we present an analysis on the characteristics of cross-tier interference in regard to femtocells deployed in LTE cellular networks. We also present a cross-tier SLNR-based water filling (CSWF) power allocation algorithm for the reduction of interference from femtocell to macrocell for smart devices used in ambient intelligence. The results of this study show that CSWF significantly improves the macro UE performance around a femtocell access point (AP) from the SINR and throughput perspective. The CSWF algorithm also provides a relative gain on the throughput of femtocell UEs compared to frequency partitioning. Furthermore, the proposed algorithm has a low complexity and is implemented on the femto-AP side only, therefore not affecting the macro system.

\section{Introduction}

As discussed in [1], statistical data shows that nearly $90 \%$ of data services and $60 \%$ of phone calls are taken in indoor environments, especially for ambient intelligence applications. New multimedia services and high data rate applications intensify the need of good quality indoor coverage [2]. Femtocell, also known as Home evolved NodeB (HeNB) in 3GPP LTE [3], uses a low power, short range (10-50 meters), low-cost, end-customer self-installed base station. Femtocells operate in a licensed spectrum owned by the mobile operator and enable Fixed Mobile Convergence (FMC) services by connecting to a cellular network via broadband communications links (e.g., DSL) [4]. The main advantage of a femtocell network is in the enhancement of indoor coverage, where the macrocell signal is weak due to wall penetration loss. Femtocells also can provide rich multimedia services to the end-customer by providing high data rate wireless access [5]. Therefore, femtocells have emerged as a promising solution to improve both the capacity and coverage in cellular networks and reduce both CPEX and OPEX. These reasons promote cellular operators to increase their revenue by investing in femtocells.

However, the mass popularization of femtocells creates several technical challenges that need to be solved. One of the major problems is cross-tier interference reduction, especially in the Closed Subscriber Group (CSG) mode introduced by 3 GPP. CSG is an access mode in which only limited and registered user equipment (UE) can access its own HeNB; any other UEs will be rejected when attempting to connect, no matter how close the distance is between them [3]. Cross-tier interference is caused by network elements that belong to different tiers or layers of the network; it may occur when operators deploy cochannel spectrum policy (i.e., $\mathrm{HeNB}$ and eNB both work under the same spectrum) [2]. For example, as shown in Figure 1, HeNB can cause downlink (DL) interference to a macrocell UE (MUE) closed to it and MUE can cause uplink (UL) interference to a neighbor 


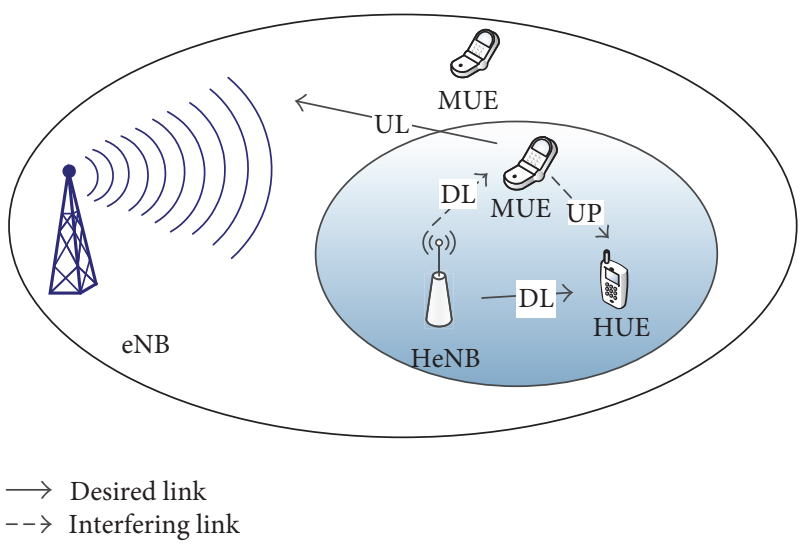

FIGURE 1: A cross-tier interference scenario between a femtocell and macrocell.

femtocell UE (FUE) when the MUE is transmitting data at high power levels. DL interference can become severe due to the fact that the DL data requirement is higher than that of the UL.

In order to cope with these kinds of interference, several schemes have been proposed in prior studies, such as power control, spectrum splitting, time splitting, and dynamic frequency partitioning. 3GPP LTE release 10 gives a frequency partitioning method which uses a central controller equipped at the eNB to allocate the spectrum for HeNB based on location information [3]. Another method found in 3GPP LTE release 10 enables the avoidance of sharing the same spectrum between eNB and near HeNB by using cell reselection priority information [3] which delivers good performance at the cell edge. Studies in [5-7] have proposed three different frequency partitioning algorithms. The main gist of them lies in that the eNB gives the control or coordinated signal directly to the HeNB via the interface $(\mathrm{X} 2, \mathrm{~S} 1)$ so that the HeNB avoids using the same spectrum. The disadvantages of frequency partitioning algorithms lie in the degradation of the frequency efficiency and scarification on the performance of the FUE, especially in dense HeNB environments. In response to these negative effects some power control algorithms have been proposed. In [8], power control is performed by HeNB equipped with cognized technology. Game theory can be considered to be an adaptive method used to deal with this problem [9-12]. 3GPP LTE also provides three different power control schemes which are based on the FUE measurement report, interference measurement from eNB, and the path loss between HeNB and MUE. However, these algorithms require cooperation with the eNB or UE, where information exchange is necessary between the HeNB and the other elements. All central control-based, information exchange-based, and signaling transmission-based algorithms will exacerbate the load of the networks. Therefore, the CPEX of the operator will increase due to the new additional functions in the eNB and UE.

In this paper, we present a power allocation (PA) algorithm in the HeNB that considers the CSG mode and cochannel spectrum policy. Cross-tier signal-to-leakage-plus-noise
(SLNR) is used in our method. There are two steps in our algorithm. Step 1 reduces the greater part of the crosstier DL interference from the HeNB. Step 2 provides a reduction of the remaining interference. Furthermore, more characteristics of this interference issue are discussed in this paper.

The remainder of this paper is organized as follows: Section 2 presents the channel and system model. Our algorithm is described in Section 3. Section 4 reports on the performance of the proposed scheme. Finally, our conclusions are given in Section 5.

\section{System and Channel Models}

In this study, the downlink of a 3GPP LTE system is considered, where the system bandwidth $B$ is divided into $N$ PRBs (Physical Resource Blocks). A PRB represents one basic time-frequency unit with a bandwidth of $180 \mathrm{kHz}$ (12 OFDM subcarriers) and a time slot of $0.5 \mathrm{~ms}$ (7 OFDM symbols). Both the eNB and HeNBs utilize the entire system bandwidth, which means that a universal frequency reuse is considered. In addition, perfect synchronizations in time and frequency are assumed.

2.1. General Definitions. We assume a given network as follows:

(i) $\mathscr{M}$ is the set of eNBs. Every eNB $m$ is at position $\vec{\rho}_{m}$ with a total transmission power of $P_{M}$, where $\vec{\rho}$ denotes the horizontal position of the network equipment. The height of all of the antennas for the eNBs is $h_{\mathrm{eNB}}$, and the transmission power of a single $\mathrm{PRB}$ is eNB specific.

(ii) $\mathscr{F}$ is the set of HeNBs. Every HeNB $f$ is at position $\vec{\rho}_{f}$ with a total transmission power of $P_{F}$.

(iii) $\mathcal{U}$ is the UE set. It is separated into two subsets which are a set of MUE $\mathcal{U}_{m}$ and a set of FUE $\mathcal{U}_{f}$. Each UE $u_{m}\left(u_{f}\right)$ is located at position $\vec{\rho}_{u}$ with a height of $h_{\mathrm{UE}}$.

(iv) $\mathcal{N}_{m(f)}$ is the set of PRBs used in eNB $m$ and HeNB $f$. Each $\operatorname{PRB} n$ has an equal transmission power for each subcarrier.

(v) $G_{m, u_{m}}^{n}\left(G_{f, u_{f}}^{n}\right)$ is the channel gain of PRB $n$ between $(\mathrm{H}) \mathrm{eNB} m(f)$ and $\mathrm{M}(\mathrm{F}) \mathrm{UE} u_{m}\left(u_{f}\right)$.

(vi) $I_{u}^{n}$ is the aggregate interference of UE $u$ composed of the eNB and HeNB interference at $\operatorname{PRB} n$.

(vii) $\eta$ is the thermal noise per PRB (including the UE noise figure).

(viii) $\gamma_{m, u_{m}}^{n}\left(\gamma_{f, u_{f}}^{n}\right)$ in (1) is the received SINR observed by $\mathrm{M}(\mathrm{F}) \mathrm{UE} u$ at $\mathrm{PRB} n$ of $(\mathrm{H}) \mathrm{eNB} m(f)$.

$$
\gamma_{m, u_{m}}^{n}=\frac{G_{m, u_{m}}^{n} p_{m}^{n}}{I_{u}^{n}+\eta}
$$


Equation (2) describes the components in the channel gain $G_{m, u_{m}}^{n}\left(G_{f, u_{f}}^{n}\right)$, which contains the antenna gain, path loss, and shadowing.

$$
\begin{aligned}
G_{m, u_{m}}^{n}\left(\vec{\rho}_{u}, \vec{\rho}_{m}, \theta, \varphi\right)= & A(\theta, \varphi)+\operatorname{PL}\left(\vec{\rho}_{u}, \vec{\rho}_{u}\right) \\
& + \text { shadowing, }
\end{aligned}
$$

where $\theta$ and $\varphi$ are the azimuth angle and elevation angle of the $\mathrm{UE}$ related to the antenna at eNB, respectively. The details of $A(\theta, \varphi), \operatorname{PL}\left(\vec{\rho}_{u}, \vec{\rho}_{m}\right)$ and shadowing will be given in Sections $2.2,2.3$, and 2.4 , respectively.

The aggregate interference $I_{u}^{n}$ definitions are described by the following:

$$
\begin{aligned}
& \text { For MUE: } I_{u_{m}}^{n}=\sum_{i \in \mathscr{M}, i \neq m} G_{i, u_{m}}^{n} p_{i}^{n}+\sum_{j \in \mathscr{F}} G_{j, u_{m}}^{n} p_{j}^{n}, \\
& \text { For FUE: } I_{u_{f}}^{n}=\sum_{i \in \mathscr{M}} G_{i, u_{f}}^{n} p_{i}^{n}+\sum_{j \in \mathscr{F}, j \neq f} G_{j, u_{f}}^{n} p_{j}^{n} .
\end{aligned}
$$

2.2. Path Loss Models. This paper considers distance based path loss models as discussed in $[13,14]$ for 3GPP LTE for three different link types.

For outdoor links (both desired and interfering) between $\mathrm{eNB}$ and MUE, the path loss is calculated using

$$
\operatorname{PL}\left(\vec{\rho}_{u}, \vec{\rho}_{m}\right)=15.3+37.6 \log _{10}\left(\left|\vec{\rho}_{u}-\vec{\rho}_{m}\right|\right) .
$$

When the MUE is located indoors, the path loss for a desired or interfering link is calculated by

$$
\operatorname{PL}\left(\vec{\rho}_{u}, \vec{\rho}_{m}\right)=15.3+37.6 \log _{10}\left(\left|\vec{\rho}_{u}-\vec{\rho}_{m}\right|\right)+20(\mathrm{~dB}) \text {. }
$$

The path loss for the desired link between HeNB and FUE and interference link between eNB (HeNB) and FUE is calculated by

$$
\operatorname{PL}\left(\vec{\rho}_{u}, \vec{\rho}_{f}\right)=127+30 \log _{10}\left(\left|\vec{\rho}_{u}-\vec{\rho}_{f}\right|\right) .
$$

This is an alternative simplified model based on the LTEA evaluation methodology which avoids modeling any walls [13]. $\left|\vec{\rho}_{u}-\vec{\rho}_{f}\right|$ is given in meters.

2.3. Shadowing. The shadowing models introduced in 3GPP [14] are applied in this paper, where all of the links implement log-normal shadowing. For the links between HeNB and the FUE served by this HeNB, the standard deviation is $4 \mathrm{~dB}$. Otherwise, for all of the other links (including interference links), the standard deviation is $8 \mathrm{~dB}$. The shadowing correlation from one UE to multiple BSs is applied, but no shadowing correlation from one BS to multiple UEs is assumed.

2.4. Antenna Patterns. In this study, the $3 \mathrm{D}$ antenna pattern given by a horizontal (azimuth) and a vertical (elevation) cut is used in order to optimize the system performance. For each sector, the $3 \mathrm{D}$ antenna pattern is described by the following:

\section{Horizontal Antenna Pattern}

$$
A_{H}\left(\varphi_{H}\right)=-\min \left[12\left(\frac{\varphi_{H}-\Phi}{\varphi_{3 \mathrm{~dB}}}\right)^{2}, A_{m}\right],
$$

TABLE 1: Link-level performance verification parameters.

\begin{tabular}{lcc}
\hline Parameter & Value & Notes \\
\hline$\alpha$, attenuation & 0.75 & Represents implementation losses \\
SINR $_{\text {Min }}, \mathrm{dB}$ & -6.5 & Based on QPSK \& 1/8 rate (DL) \\
SINR $_{\text {Max }}, \mathrm{dB}$ & 17 & Based on 64QAM \& 4/5 rate (DL) \\
$\mathrm{Thr}_{\text {Max }}, \mathrm{bps} / \mathrm{Hz}$ & 4.8 & Based on 64QAM \& 4/5 rate (DL) \\
\hline
\end{tabular}

where $A_{H}$ is the horizontal antenna pattern, $\Phi$ is the azimuth orientation, $\varphi_{3 \mathrm{~dB}}$ is the angle of the $3 \mathrm{~dB}$ antenna gain (beamwidth), and $A_{m}$ is the backward attenuation. Typical values for these parameters are $\varphi_{3 \mathrm{~dB}}=70^{\circ}, A_{m}=20 \mathrm{~dB}$, and $\Phi=\left\{0,120^{\circ},-120^{\circ}\right\}$.

\section{Vertical Antenna Pattern}

$$
A_{V}\left(\theta_{V}\right)=-\min \left[12\left(\frac{\theta_{V}-\theta_{\text {etilt }}}{\theta_{3 \mathrm{~dB}}}\right)^{2}, \mathrm{SLA}_{v}\right],
$$

where $A_{V}$ is the vertical antenna pattern, $\theta_{\text {etilt }}$ is the electrical antenna downtilt, $\theta_{3 \mathrm{~dB}}$ is the angle of the $3 \mathrm{~dB}$ antenna gain (elevation beamwidth), and SLA $v$ is the side lobe attenuation. Typical values for these parameters are $\theta_{3 \mathrm{~dB}}=10^{\circ}, \mathrm{SLA}_{v}=$ $20 \mathrm{~dB}$, and $\theta_{\text {etilt }}=\left(5^{\circ}, 15^{\circ}\right)$. Therefore, the $3 \mathrm{D}$ antenna pattern can be defined as

$$
A(\theta, \varphi)=-\min \left[A(\theta)_{V}+A(\varphi)_{H}, A_{m}\right]
$$

The definitions of the angles $\varphi_{H}$ and $\theta_{V}$ are straightforward:

$$
\begin{aligned}
\varphi_{H} & =\angle\left(\vec{\rho}_{u}, \vec{\rho}_{m}\right) ; \\
\theta_{V} & =\arctan \left(\frac{h_{\mathrm{eNB}}-h_{\mathrm{UE}}}{\left|\vec{\rho}_{u}-\vec{\rho}_{m}\right|}\right) .
\end{aligned}
$$

The azimuth antenna patterns for all of the UEs and HeNBs are assumed to be omnidirectional [13].

2.5. Link Adaptation. Link adaptation is implemented where the used modulation and coding scheme are selected based on the received SINR. In order to model the link adaptation, the SINR is mapped to the throughput using the attenuated and truncated Shannon bound method described in the 3GPP standard [15]. Given a particular SINR $\gamma_{m, u}$ the throughput on the $\mathrm{PRB} n$ for $\mathrm{UE} u$ is determined by

$$
\begin{aligned}
& \text { Thr (bps/Hz) } \\
& = \begin{cases}0, & \text { SINR }<\mathrm{SINR}_{\mathrm{Min}} \\
\alpha \cdot S(\mathrm{SINR}), & \text { SINR }_{\text {Min }}<\mathrm{SINR}_{\mathrm{SIN}}<\mathrm{SINR}_{\text {Max }} \\
\mathrm{Thr}_{\mathrm{Max}}, & \text { SINR } \mathrm{SINR}_{\mathrm{Max}},\end{cases}
\end{aligned}
$$

where the Shannon bound is $S\left(\gamma_{m, u}\right)=\log _{2}\left(1+\gamma_{m, u}\right)$ in bps/Hz. The other parameters are summarized in Table 1 $[6,15]$. 


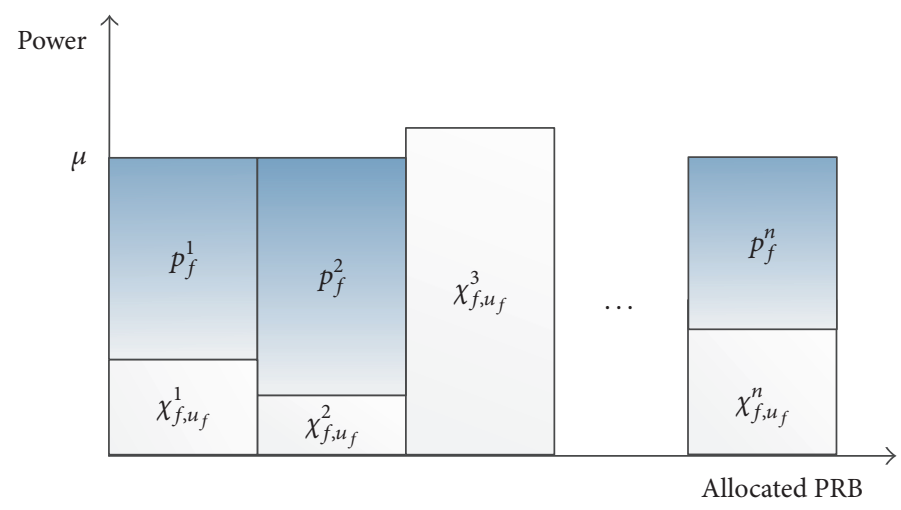

FIgure 2: An example of CSWF.

\section{Two-Step PA for Cross-Tier Interference Reduction}

3.1. DL Interference in LTE Macro-Femto Network. Interference management in two-tier networks faces a practical challenge from the lack of coordination between the eNBs and HeNBs due to scalability, security, and the limited availability of backhaul bandwidth $[9,16]$. From an infrastructure or spectrum availability perspective, it may be easier to operate the eNBs and HeNBs in a common spectrum; at the same time, pragmatic solutions are necessary in order to reduce cross-tier interference $[9,17]$.

Our work assumes that a Closed Subscriber Group (CSG) is being used, which means only licensed UEs within radio range can access their HeNBs. With FUEs in CSG, cross-tier interference from indoor HeNBs may significantly deteriorate the SINR for the MUEs. The prerequisite of our proposal is to ensure that the MUE performance remains unaffected by coexisting HeNBs operating in the same frequency band. That is, the legacy system, the 3GPP LTE eNB system used in this paper, is a primary infrastructure used to ensure that MUE achieves its minimum SINR target to its eNB, even though indoor CSG users act in their own self-interest to maximize their SINRs generating cross-tier femtocell interference [9].

Since the eNB transmission power is much greater than the HeNB transmission power in most cases, the interference suffered by MUE will be dominated by the neighboring eNB interference. However, only if MUE is located close to $\mathrm{HeNB}$, it will be exposed to high HeNB interference. For the worst case when MUE is located indoors alongside HeNB, it will be likely to suffer from poor SINR due to the small channel gain caused by wall penetration loss and the high interference from the HeNB. By universal frequency reuse, HeNBs utilize all available $\mathrm{PRBs}$, which results in unacceptable SINR degradation to the MUE closed to the HeNB irrespective of the use of any available PRBs.

\subsection{The Proposed Cross-Tier SLNR-Based PA in HeNB}

Step 1. The SLNR concept is introduced in [18-20] in order to improve the performance of cell edge users. In these studies, the SLNR is adopted in the systems employing MIMO (multiple input multiple output) techniques, such as antenna selection, precoding, and beamforming.

Since the SLNR deals with the relationship between desired link and interfering link, it is an appropriate criterion to be employed for interference reduction. In this paper, we define cross-tier SLNR of HeNB as

$$
\kappa_{f, u_{f}}^{n}=\frac{p_{f}^{n} G_{f, u_{f}}^{n}}{\sum_{i \in \mathrm{M}} G_{i, u_{f}}^{n} p_{i}^{n}+\eta} .
$$

The numerator of (12) denotes the received signal power at the FUE; the denominator denotes the received interference power from HeNB $f$ plus the noise at the MUEs. Transmission power allocation is performed at each HeNB to maximize the SLNR metric for each active FUE.

In order to determine the transmission power of each PRB for HeNB, a modified water filling (WF) power allocation algorithm, entitled the Cross-tier SLNR-based Water Filling (CSWF) method, is presented in this paper. It is well known that the conventional WF theorem [21] provides an optimum solution for power allocation in a parallel Gaussian channel by using the received SNR. However, the proposed CSWF incorporates the cross-tier SLNR, instead of the SNR used in the conventional WF, in order to generate the water bottom shown in Figure 2. In the CSWF method, $\chi_{f, u_{f}}^{n}$ is considered to be the water bottom and is defined as

$$
\chi_{f, u_{f}}^{n}=\frac{\sum_{i \in \mathrm{M}} G_{i, u_{f}}^{n} p_{i}^{n}+\eta}{G_{f, u_{f}}^{n}} .
$$

By using the CSWF, the optimum power for each PRB, that is, the transmit power of $\operatorname{HeNB} f$ at $\operatorname{PRB} n$, is determined by

$$
p_{f}^{n}= \begin{cases}\mu-\chi_{f, u_{f}}^{n}, & \chi_{f, u_{f}}^{n}<\mu, \\ 0, & \chi_{f, u_{f}}^{n} \geq \mu .\end{cases}
$$

Here, $\mu$ is the so-called water level and is chosen so that

$$
\sum_{n}^{N}\left(\mu-p_{f}^{n}\right)^{+}=P_{F}
$$




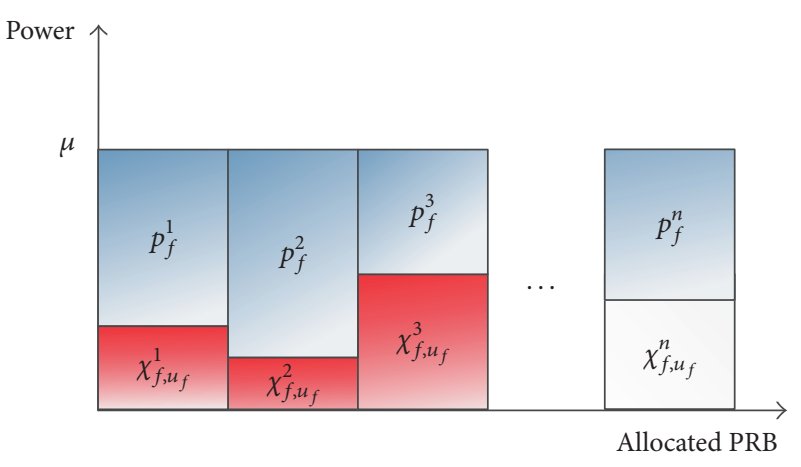

(a) Power allocation in HeNB without interference reduction

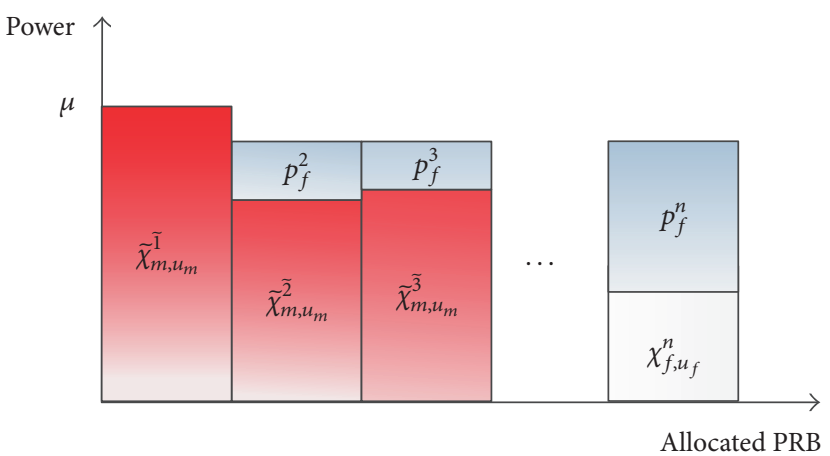

(b) Power allocation in HeNB with interference reduction

FIGURE 3: Interference reduction based on the CSI of the MUE.

where

$$
x^{+}= \begin{cases}x, & x>0 \\ 0, & x \leq 0 .\end{cases}
$$

The transmission power allocated by the CSWF method at the HeNB not only maximizes the received power at the FUE, but also simultaneously minimizes the interference to the MUE. However, in severe interference environments where the MUE is located indoors near the HeNB, as mentioned in Section 3.1, the received SINR at the MUE might be still unacceptable even after applying Step 1 of the CSWF; in other words it is lower than the minimum received SINR requirement of the link adaptation outlined in Section 2.4, making it beneficial to provide further interference reduction at the HeNB.

Step 2. By keeping in mind the problems mentioned above, we present a cross-tier interference reduction algorithm based on the channel state information (CSI) of the MUE in the downlink (DL). Since HeNB serves only one or two FUEs in practice, each FUE has more available PRBs compared to the MUE. Furthermore, FUE always has a better channel status than MUE. Therefore, the reduction of the HeNB transmission power will not significantly affect the FUE and will provide better performance to the MUE.

The main concept of our algorithm in Step 2 is to perform CSWF at the HeNB, where the water bottoms are generated by the SINR of the MUE (based on the CSI report from MUEs to eNB) instead of the SLNR of the FUE on overlapped PRBs shared by the FUE and the adjacent MUEs, since the HeNB needs to limit the transmission power level on those PRBs in order to reduce the interference level from the femtocell to the MUEs. As illustrated in Figure 3, we use $\widetilde{\chi}_{m, u_{m}}^{\widetilde{n}}$ to take the place of $\chi_{f, u_{f}}^{n}$ :

$$
\tilde{\chi}_{m, u_{m}}^{\tilde{n}}=\frac{\sum_{i \in M, i \neq m} G_{i, u_{m}}^{\tilde{n}} p_{i}^{\tilde{n}}+\sum_{j \in \mathrm{F}} G_{j, u_{m}}^{\tilde{n}} p_{j}^{\tilde{n}}+\eta}{G_{m, u_{m}}^{\tilde{n}}},
$$

where $\widetilde{n}$ is the index of the limited PRBs. Comparing $\widetilde{\chi}_{m, u_{m}}^{\widetilde{n}}$ to $\chi_{f, u_{f}}^{n}, G_{m, u_{m}}^{\tilde{n}}$ is smaller than $G_{f, u_{f}}^{n}$ due to the path loss and the penetration loss. $\sum_{i \in \mathbb{M}, i \neq m} G_{i, u_{m}}^{\tilde{n}} p_{i}^{\tilde{n}}+\sum_{j \in \mathrm{F}} G_{j, u_{m}}^{\tilde{n}} p_{j}^{\tilde{n}}$ (the cross-tier interference plus the intercell interference) is larger than $\sum_{i \in \mathrm{M}} G_{i, u_{f}}^{n} p_{i}^{n}$ (the cross-tier interference), because the prominent part of the interference from nearby HeNB is considered in both, and the cross-tier plus the intercell interference also contains the interference from the eNBs which is higher than the other interferences. As a result $\tilde{\chi}_{m, u_{m}}^{\tilde{n}}$ is much larger than $\chi_{f, u_{f}}^{n}$. By using $\widetilde{\chi}_{m, u_{m}}^{\tilde{n}}$ the CSWF will reallocate less power in the shared PRBs. In other words, the interference will be degraded.

The transmission power of $\mathrm{HeNB}(f)$ at $\mathrm{PRB} n$ will be changed according to $\widetilde{\chi}_{m, u_{m}}^{\tilde{n}}$ :

$$
p_{f}^{n}= \begin{cases}\mu-\chi_{f, u_{f}}^{n}, & \chi_{f, u_{f}}^{n}<\mu, \text { for } n \\ \mu-\widetilde{\chi}_{m, u_{m}}^{\tilde{n}}, & \widetilde{\chi}_{m, u_{m}}^{\widetilde{n}}<\mu, \text { for } \widetilde{n} \\ 0, & \chi_{f, u_{f}}^{n}\left(\widetilde{\chi}_{m, u_{m}}^{\tilde{n}}\right) \geq \mu .\end{cases}
$$

The proposed algorithm can be easily understood by referring to Figure 3, in which part (a) illustrates the normal power allocation (described in Section 3.2) in HeNB. The red area indicates that these PRBs are used by both the femto and macro users the same time. Figure 3(b) clearly shows that the proposed power allocation algorithm applies less power to the overlapped PRBs. The change in the SINR at the MUE can be observed in Figure 4.

The amount of interference reduced by using the proposed algorithm can be mathematically analyzed as follows: Define $p_{f}^{\widetilde{n}}$ and $\widetilde{p}_{f}^{\widetilde{n}}$ to be the transmission powers before and after the interference reduction, respectively; hence the reduced power is calculated by using

$$
\begin{aligned}
\Delta p_{f}^{\tilde{n}} & =p_{f}^{\tilde{n}}-\widetilde{p}_{f}^{\widetilde{n}}=\left(\mu-\chi_{f, u_{f}}^{n}\right)-\left(\mu-\tilde{\chi}_{m, u_{m}}^{\tilde{n}}\right) \\
& =\widetilde{\chi}_{m, u_{m}}^{\tilde{n}}-\chi_{f, u_{f}}^{n} .
\end{aligned}
$$

The interference is decreased by a substantial amount and is computed by

$$
\Delta I_{f}^{\tilde{n}}=G_{f, u_{m}}^{\tilde{n}}\left(\tilde{\chi}_{m, u_{m}}^{\tilde{n}}-\chi_{f, u_{f}}^{n}\right) .
$$




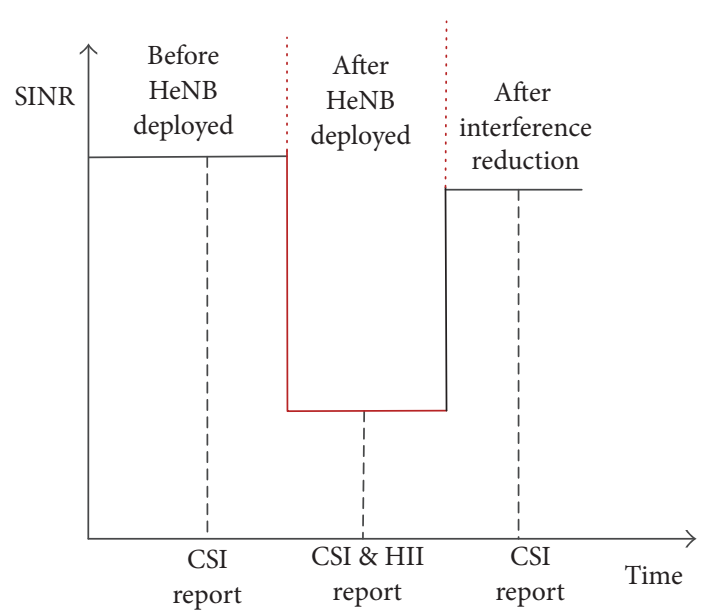

FIGURE 4: Improvement of the SINR after employing CSWF Step 2.

The equation used to calculate the improved SINR is

$$
\Delta \gamma_{m, u_{m}}^{\widetilde{n}}=G_{m, u_{m}}^{\tilde{n}} p_{m}^{\tilde{n}}\left(\frac{1}{I_{u}^{\tilde{n}}-\Delta I_{f}^{\tilde{n}}+\eta}-\frac{1}{I_{u}^{\tilde{n}}+\eta}\right) .
$$

The simulation results in Section 4 show that our implemented algorithm outperformed the conventional methods by obtaining a remarkable increase in the SINR.

In a practical implementation of Step 2, the CSI report from the MUEs to the eNB is used for resource allocation for the eNB and HeNB. If the MUE is suffering from a high interference larger than the preset threshold, it will report a CSI of poor SINR and high interference indicator (HII) to the eNB, as shown in Figure 4. This CSI report can be utilized as the reference to tell the HeNB to reduce the transmission power.

There are three advantages provided by this algorithm:

(1) The SINR information of MUE is easy to detect.

(2) $\tilde{\chi}_{m, u_{m}}^{\tilde{n}}$ can be directly utilized in the CSWF without any other processing.

(3) $\tilde{\chi}_{m, u_{m}}^{\tilde{n}}$ is MUE-specific; hence, the CSWF can reallocate power dynamically and specifically to different MUEs.

3.3. Implementation in LTE Femtocell System. It should be noted that a DL receiver should be a part of HeNB. DL receiver can detect and receive the DL control signal from the eNB to the MUEs. This DL receiver function can also be called the Network Listen Mode (NLM), Radio Environment Measurement (REM), or "HeNB Sniffer" [3]. At the same time, measurement, detection, and receiving of the UL signal (feedback report) from the MUEs to the eNB can be done by the UL receiver [3]. The main procedure of our algorithm operated by $\mathrm{HeNB}$ is the following:

(1) Use the HeNB UL receiver to detect the signal strength from the surrounding MUEs:

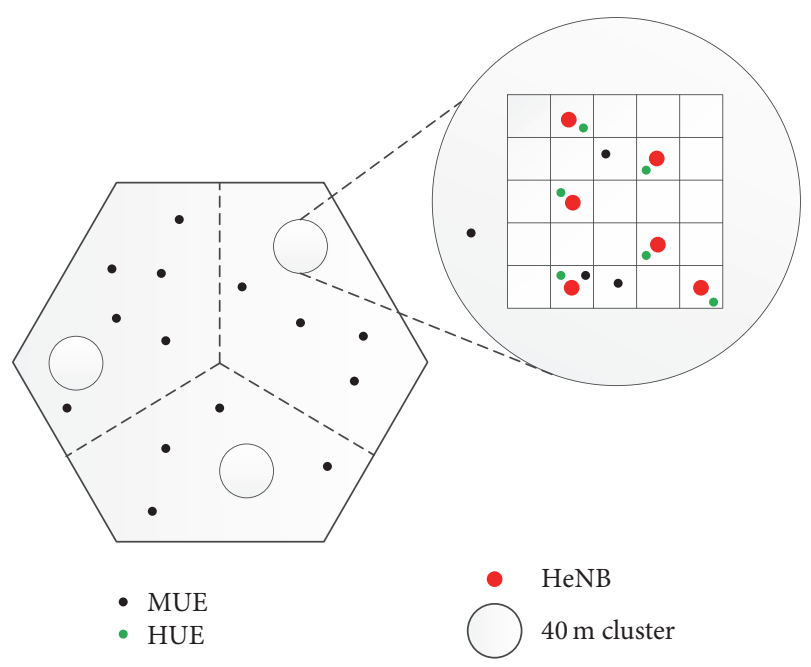

FIGURE 5: Cell layout for performance evaluation.

(i) To determine the MUEs which are suffering potential high interference

(ii) To detect the cross-tier SLNR of the HeNB

(2) Use the HeNB DL receiver to receive the control information from eNB to MUEs:

(i) To capture the resource management information (e.g., PRB allocation and time slot) of the adjacent MUEs

(3) The HeNB allocates power to each PRB in the FUE based on CSWF Step 1.

(4) Use the HeNB UL receiver to receive the HII reported by interfering MUEs:

(i) Based on the HII, to pick up resource management information of the interfering MUEs found in (2)

(5) Use HeNB UL receiver to receive the channel state information from the interfering MUEs.

(6) Power reallocation at the HeNB is based on CSWF Step 2:

(i) To reduce the transmit power at a shared PRB due to a bad channel state

\section{SLS Performance Verification}

4.1. Cell Layout and Parameters Setup. As shown in Figure 5, 19 regular hexagonal cells with an intersite distance of $500 \mathrm{~m}$ were assumed. Every cell contained three sectors served by the same eNB. In Figure 5, a $40 \mathrm{~m}$ cluster was in each macro sector, and we posited a single-floor building with 25 apartments in each cluster. The apartments were $10 \mathrm{~m} \times 10 \mathrm{~m}$ and placed next to each other in a $5 \times 5$ grid $[13,14]$. Some macro UEs (MUEs) were placed randomly in the clusters 
TABLE 2: SLS parameters.

\begin{tabular}{lc}
\hline Parameter & Value \\
\hline Inter-eNB distance & $500 \mathrm{~m}$ \\
Sectors per eNB & 3 \\
Num. of active MUE per sector & 10 \\
Num. of buildings in one cell & 1 \\
Num. of active HeNB in one building & 6 \\
FUE per HeNB & 1 \\
Minimum distance between UE and eNB & $35 \mathrm{~m}$ \\
Minimum distance between UE and HeNB & $20 \mathrm{~cm}$ \\
Height of eNB & $32 \mathrm{~m}$ \\
Height of UE & $1.5 \mathrm{~m}$ \\
Frequency reuse & 1 \\
Bandwidth & $20 \mathrm{MHz}$ \\
Tot. num. of available PRBs & 100 \\
PRB bandwidth & $180 \mathrm{kHz}$ \\
Thermal noise & $-174 \mathrm{dBm} / \mathrm{Hz}$ \\
eNB/HeNB antenna gain & $14 \mathrm{dBi} / 0 \mathrm{dBi}$ \\
Tot. Tx power of eNB/HeNB & $49 \mathrm{dBm} / 20 \mathrm{dBm}$ \\
UE noise figure & $9 \mathrm{~dB}$ \\
UE Rx antenna & 1 \\
eNB/HeNB Tx antenna & 1 \\
Traffic model & Full buffer \\
\hline
\end{tabular}

(35\% in this exercise); the remaining MUEs were located within the cell area. We deployed $6 \mathrm{HeNBs}$ in each grid; each HeNB served only one FUE randomly located in the corresponding apartment.

In our system-level simulation (SLS), the exact same parameters given in $[13,14]$ were adopted as common parameters. These parameters are summarized in Table 2.

4.2. SLS Results. Figure 6 demonstrates the desirability and benefits of interference reduction at the HeNB. The cumulative distribution function (CDF) of the DL SINR for the MUEs with and without the CSWF algorithm is shown in Figure 6. Due to the nonuniform distribution of the MUEs, which means a fixed percentage of MUEs must be located in clusters, the SINR curves are not smooth. Based on link adaptation, there are two important SINR points, $-6.5 \mathrm{~dB}$ and $17 \mathrm{~dB}$, which indicate the minimum connection requirement (i.e., the throughput equal to zero) and maximum throughput requirement. The green curve represents the situation where no HeNB was deployed (ideal case: upper bound of performance). The black solid line represents the situation where the HeNB was deployed but without utilizing any interference management (worst case: lower bound of performance). From the figure, it is clearly evident that by deploying the HeNB without interference management the SINR degrades around $23.5 \mathrm{~dB}$ at a CDF of $40 \%$ compared to the upper bound. The red line depicts the scenario where the interference is reduced by the PRB partitioning algorithm, which has been proposed as an effective way to mitigate the interference from the HeNB. The dashed blue line depicts the scenario where the interference is only reduced by
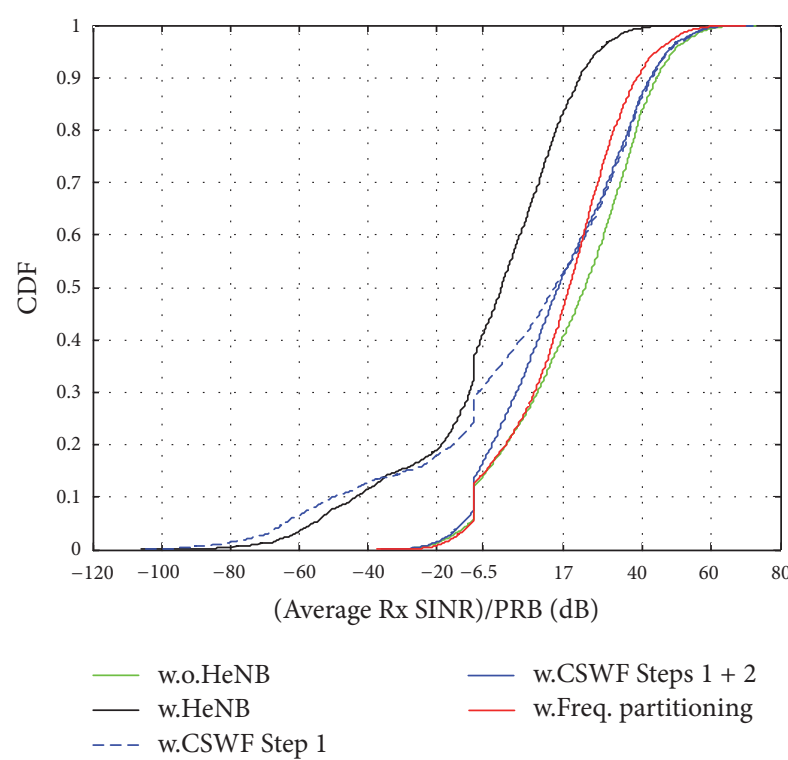

- w.CSWF Steps $1+2$

- w.Freq. partitioning

FIgURE 6: CDF of the average Rx SINR per PRBs at the MUEs.

CSWF Step 1; the solid blue line depicts the combination of Steps $1+2$. It can be observed that the CSWF algorithm remarkably improves the SINR performance. At the SINR level of $-6.5 \mathrm{~dB}$, the CSWF protects more than $20 \%$ of the PRBs from disconnection, and the gap between the CSWF and upper bound is less than $2.5 \%$. At the SINR level of $17 \mathrm{~dB}$, compared to the lower bound, the CSWF algorithm enhances the performance of around $30 \%$ of the PRBs. The frequency partitioning algorithm gives a better performance below the CDF value of 0.6 , because it does not use any overlapped frequencies that can cause high levels of interference (larger than threshold). However, our proposed CSWF algorithm performs better, when the CDF value is larger than 0.6, due to the fact that the CSWF algorithm reduces the interference to all of the adjacent MUEs (victims) in Step 1.

Figure 7 shows the performance of the average throughput given by one PRB. Due to link adaptation, the throughput does not increase after $864 \mathrm{kbps}$ (64QAM with code rate of $4 / 5)$. It is clear that without any interference management about $41 \%$ of the PRBs are wasted. This is due to the severe cross-tier interference caused by the deployment of the HeNB. On the other hand, when the CSWF algorithm is applied, a meaningful throughput gain can be achieved. At the CDF of 0.5 , the simulation results for the CSWF show a nearly $0.58 \mathrm{Mbps} / \mathrm{PRB}$ gain, and the percentage of wasted PRBs decreases from $41 \%$ to $16 \%$. However, we can note one interesting phenomenon from Figure 7 in that the average CSWF throughput is always worse than that of frequency partitioning, even though Figure 6 shows that the proposed CSWF algorithm outperforms frequency partitioning. The reason is that by using the link adaptation given in (11) the maximum throughput can be achieved at the SINR of $17 \mathrm{~dB}$. But, from Figure 6, we can see that our proposed CSWF performs better than frequency partitioning after the SINR reaches $20 \mathrm{~dB}$. Therefore, in the foreseeable future, higher order modulation schemes will be included in the 


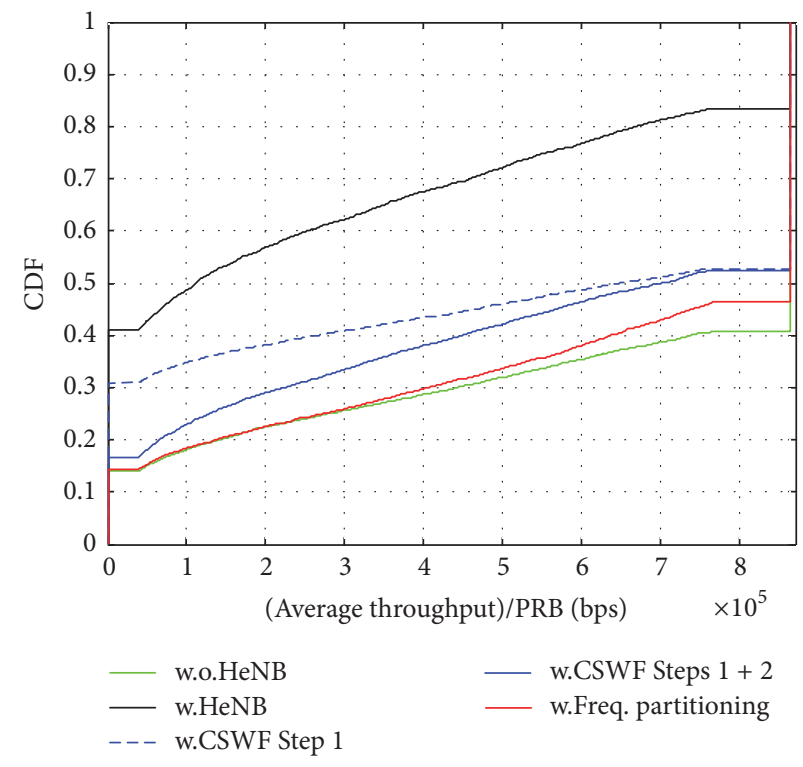

FIGURE 7: CDF of the average throughput per PRBs at the MUEs.

TABLE 3: MUE average throughput.

\begin{tabular}{lcc}
\hline & Macro UE average throughput (bps) & Throughput loss ratio \\
\hline Without HeNB & 6098932 & 0 \\
Without interference management & 2830337 & $53.6 \%$ \\
CSWF Step 1 & 4853119 & $20.4 \%$ \\
CSWF Steps 1 + & 5340644 & $12.4 \%$ \\
Freq. partitioning & 5927775 & $2.8 \%$ \\
\hline
\end{tabular}

standards. That is, a higher SINR (corresponding to higher order modulation schemes) gives benefits, since the CSWF algorithm performs better than frequency partitioning in the high SNR range.

Figures 6 and 7 also reveal the relationship between CSWF Steps 1 and 2. In Figure 6, it can be observed that the dashed and solid blue lines overlap after a CDF of 0.5 , which implies that the CSWF Step 1 is the primary contributor of this part. Below a CDF of 0.5, the integrated CSWF shows a gap compared to just utilizing Step 1, which implies that remaining severe interference is reduced by Step 2 .

Table 3 summarizes the throughput loss of different interference management scenarios, with the HeNB deployment compared to the scenario without HeNB. By applying CSWF the throughput loss improves from $53.6 \%$ to $12.4 \%$. Compared to the PRB partitioning algorithm, the CSWF algorithm performs 7.6\% worse. However, in Table 4 we can see that the CSWF only generates $1.1 \%$ degradation of the FUE average throughput compared to the scenario where no interference management algorithm is applied. In this case, the frequency partitioning algorithm degrades the average throughput of the FUE by about $16.1 \%$. Table 5 reveals the benefits of deploying HeNBs. By deploying HeNB and applying the CSWF algorithm, the system spectrum efficiency improves by more than 8 times compared to the case of an eNB only network. Above data analyses are presented for formalisms and methods the same as in $[22,23]$.

\section{Conclusions}

In this paper, a power allocation algorithm for cross-tier interference reduction in LTE macro-femto coexisting systems has been presented for use in ambient intelligence applications. Based on the simulation results, we conclude that the interference from $\mathrm{HeNB}$ can be remarkably reduced, showing a $10 \mathrm{~dB}$ improvement on the Rx SINR of the MUEs by applying the CSWF algorithm. A system employing CSWF doubles the MUE throughput compared to one having no interference management. Furthermore, the CSWF algorithm only degrades the throughput of the FUE by $1.1 \%$, which is almost negligible compared with the $16.1 \%$ generated by frequency partitioning. In addition, CSWF is a selforganized algorithm, and so no coordination and signaling exchange between the HeNB and eNB are necessary. Since HeNBs are self-setup by the customer and the operator does not want any modification on eNB and UE, we believe that distributed and self-operating algorithms like CSWF at the 
TABLE 4: FUE average throughput.

\begin{tabular}{lcc}
\hline & HeNB UE average throughput (bps) & Throughput loss ratio \\
\hline Without interference management & 512156730 & 0 \\
CSWF & 461508050 & $1.1 \%$ \\
Freq. partitioning & 429910091 & $16.1 \%$ \\
\hline
\end{tabular}

TABLE 5: System spectrum efficiency (over $20 \mathrm{MHz}$ ).

\begin{tabular}{lc}
\hline & Spectrum efficiency $(\mathrm{bps} / \mathrm{Hz})$ \\
\hline Without HeNB & 3.05 \\
CSWF Steps 1 + & 25.75 \\
Freq. partitioning & 24.54 \\
\hline
\end{tabular}

femtocell side regarding interference reduction are more promising than other central control or coordinated algorithms. In the future work, we will apply our proposed scheme dedicated to $5 \mathrm{G}$ telecommunications, for example, under the consideration of massive MIMO and nonorthogonal multiple access (NOMA).

\section{Competing Interests}

The authors declare that they have no competing interests.

\section{Acknowledgments}

This research was supported by Basic Science Research Program through the National Research Foundation of Korea (NRF) funded by the Ministry of Science, ICT \& Future Planning (2015R1C1A1A02037515). This research was also supported by the Natural Science Foundation of Jiangsu Province under Grant BK20160287 and in part by the Fundamental Research Funds for the Central Universities under Grant 2015B30614.

\section{References}

[1] G. Mansfield, "Femtocells in the US market-business drivers and consumer proposition," in Proceedings of the Femtocells Europe, London, UK, June 2008.

[2] T. Zahir, K. Arshad, A. Nakata, and K. Moessner, "Interference management in femtocells," IEEE Communications Surveys and Tutorials, vol. 15, no. 1, pp. 293-311, 2013.

[3] 3GPP, "Home eNode B(HeNB) Radio Frequency (RF) requirements analysis," 3GPP TR 36.921, V 10.0.0, 2011.

[4] V. Chandrasekhar, J. G. Andrews, and A. Gatherer, "Femtocell networks: a survey," IEEE Communications Magazine, vol. 46, no. 9, pp. 59-67, 2008.

[5] D. Klaus, M. Martti, and V. Kimmo, "On interference management for uncoordinated LTE-Femto cell deployments," in Proceedings of the 11th European Wireless Conference 2011Sustainable Wireless Technologies (European Wireless), pp. 1-6, April 2011.

[6] Z. Bharucha, A. Saul, G. Auer, and H. Haas, "Dynamic resource partitioning for downlink femto-to-macro-cell interference avoidance," EURASIP Journal on Wireless Communications and Networking, vol. 2010, Article ID 143413, pp. 1-12, 2010.

[7] Z. Zhao, F. Zheng, A. Wilzeck, and T. Kaiser, "Femtocell spectrum access underlaid in fractional frequency reused macrocell," in Proceedings of the IEEE International Conference on Communications Workshops (ICC '11), June 2011.

[8] S.-M. Cheng, W. C. Ao, and K.-C. Chen, "Downlink capacity of two-tier cognitive femto networks," in Proceedings of the IEEE 21st International Symposium on Personal Indoor and Mobile Radio Communications (PIMRC '10), pp. 1303-1308, Istanbul, Turkey, September 2010.

[9] V. Chandrasekhar, J. G. Andrews, T. Muharemovic, Z. Shen, and A. Gatherer, "Power control in two-tier femtocell networks," IEEE Transactions on Wireless Communications, vol. 8, no. 8, pp. 4316-4328, 2009.

[10] J.-H. Yun and K. G. Shin, "Adaptive interference management of OFDMA femtocells for co-channel deployment," IEEE Journal on Selected Areas in Communications, vol. 29, no. 6, pp. 12251241, 2011.

[11] X. Kang, Y.-C. Liang, and H. K. Garg, "Distributed power control for spectrum-sharing femtocell networks using Stackelberg game," in Proceedings of the IEEE International Conference on Communications (ICC '11), pp. 1-6, Kyoto, Japan, June 2011.

[12] J.-S. Lin and K.-T. Feng, "Game theoretical model and existence of win-win situation for femtocell networks," in Proceedings of the IEEE International Conference on Communications (ICC '11), pp. 1-6, IEEE, Kyoto, Japan, June 2011.

[13] 3GPP, "Further advancements for E-UTRA physical layer aspects," 3GPP TR 36.814, V 9.0.0, 2010.

[14] 3GPP, "Simulation assumptions and parameters for FDD HeNB RF requirements," Tech. Rep. 3GPP R4-091422, 2009.

[15] 3GPP, "Evolved Universal Terrestrial Radio Access (E-UTRA); Radio Frequency (RF) system scenarios," 3GPP TR 36.942, V 10.2.0, 2010.

[16] A. Zemlianov and G. De Veciana, "Cooperation and decisionmaking in a wireless multi-provider setting," in Proceedings of the IEEE Conference on Computer Communications (INFOCOM "05), vol. 1, pp. 386-397, Miami, Fla, USA, March 2005.

[17] H. Claussen, "Performance of macro- and co-channel femtocells in a hierarchical cell structure," in Proceedings of the 18th Annual IEEE International Symposium on Personal, Indoor and Mobile Radio Communications( PIMRC '07), IEEE, Athens, Greece, September 2007.

[18] A. Tarighat, M. Sadek, and A. H. Sayed, "A multi user beamforming scheme for downlink mimo channels based on maximizing signal-to-leakage ratios," in Proceedings of the IEEE International Conference on Acoustics, Speech, and Signal Processing (ICASSP '05), vol. 3, pp. 1129-1132, IEEE, March 2005.

[19] M. Sadek, A. Tarighat, and A. H. Sayed, "A leakage-based precoding scheme for downlink multi-user MIMO channels," IEEE Transactions on Wireless Communications, vol. 6, no. 5, pp. 17111721, 2007. 
[20] M. Sadek, A. Tarighat, and A. H. Sayed, "Active antenna selection in multiuser MIMO communications," IEEE Transactions on Signal Processing, vol. 55, no. 4, pp. 1498-1510, 2007.

[21] T. M. Cover and J. A. Thomas, Elements of Information Theory, John Wiley \& Sons, Hoboken, NJ, USA, 2nd edition, 2005.

[22] L. Ogiela, "Cognitive computational intelligence in medical pattern semantic understanding," in Proceedings of the 4th International Conference on Natural Computation (ICNC '08), M. Z. Guo, L. Zhao, and L. P. Wang, Eds., vol. 6, pp. 245-247, Jian, China, October 2008.

[23] L. Ogiela and M. R. Ogiela, "Data mining and semantic inference in cognitive systems," in Proceedings of the International Conference on Intelligent Networking and Collaborative Systems (IEEE INCoS '14), F. Xhafa, L. Barolli, F. Palmieri et al., Eds., pp. 257-261, Salerno, Italy, September 2014. 

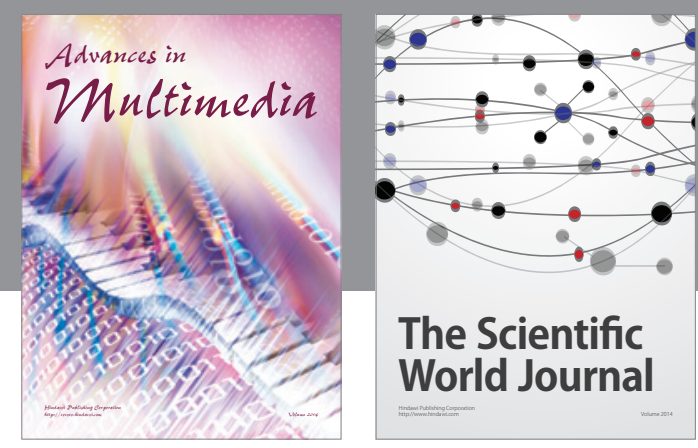

The Scientific World Journal
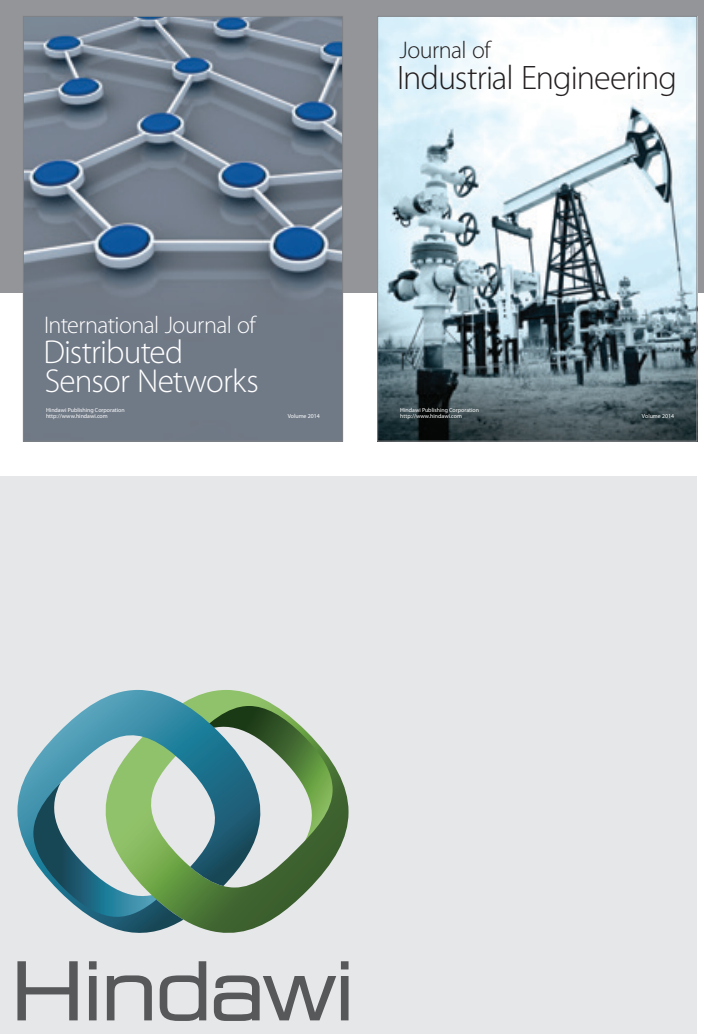

Submit your manuscripts at

http://www.hindawi.com

\section{Computer Networks} and Communications
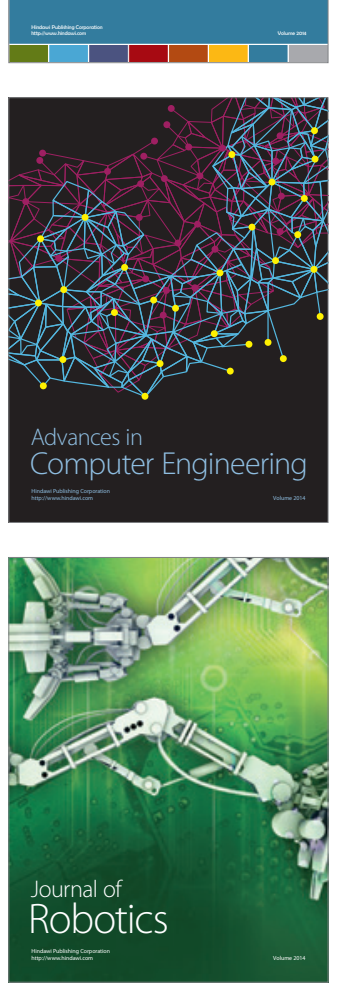
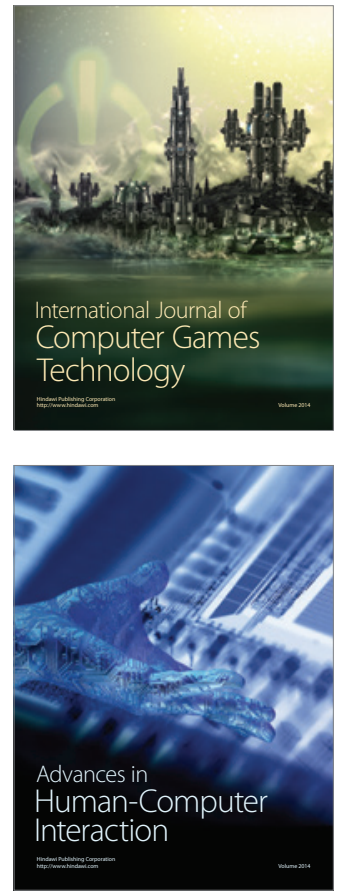
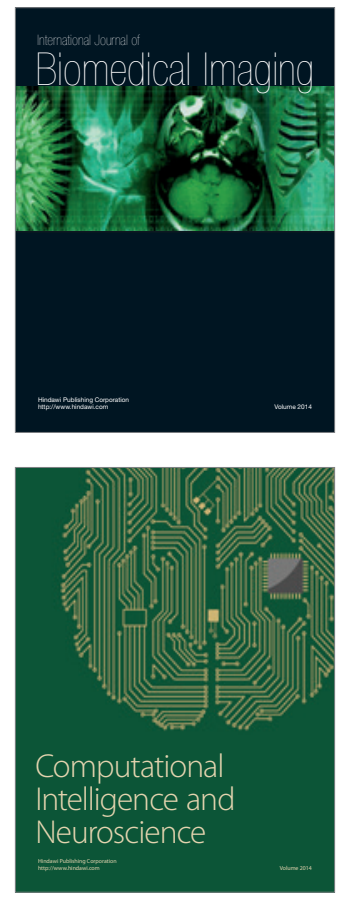
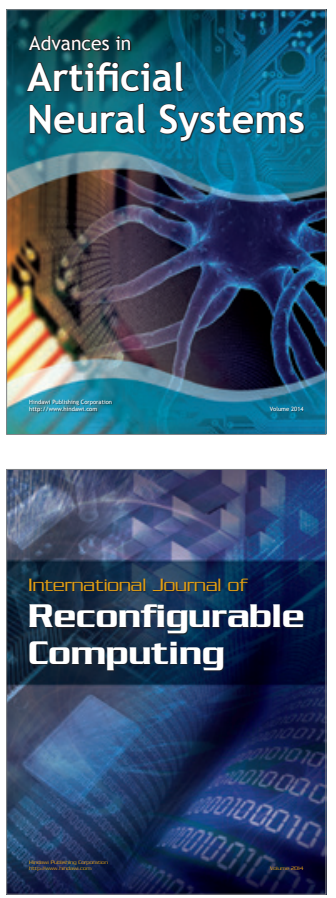
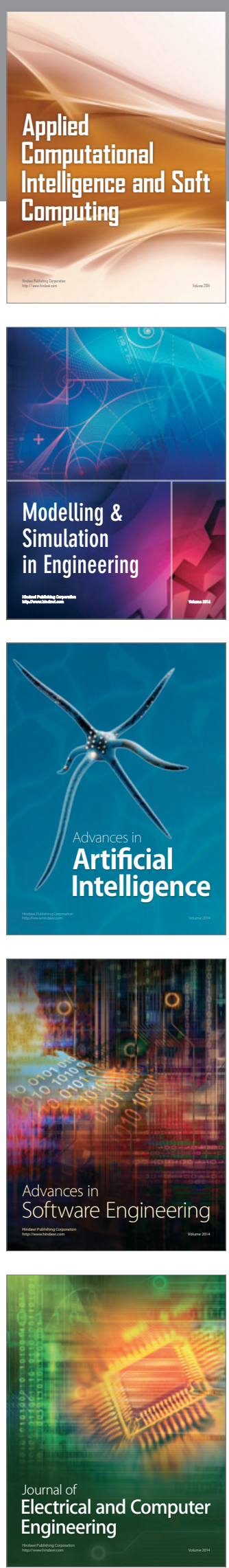